\title{
Enraizamento de estacas e produção de Euryops chrysanthemoides (DC.) B. Nord. em vaso ${ }^{(1)}$
}

\author{
JANINE FARIAS MENEGAES(2)*, FERNANDA ALICE ANTONELLO LONDERO BACKES(2), \\ ROGÉRIO ANTÔNIO BELLÉ( ${ }^{(2)}$ E TÚLIO EDUARDO DE SEIXAS PERES ${ }^{(3)}$
}

\begin{abstract}
RESUMO
Os experimentos foram conduzidos no Setor de Floricultura da UFSM no período de agosto de 2013 a maio de 2014, em duas fases experimentais. O primeiro experimento teve o objetivo de verificar o enraizamento de estacas de Euryops chrysanthemoides em diferentes concentrações de ácido indolbutírico (AIB), foi utilizado o delineamento blocos casualizados, avaliou-se o comprimento e o número de raízes. No segundo experimento avaliou-se diferentes densidades de plantas de E. chrysanthemoides em vaso (1, 2, 3 ou 4 plantas), em delineamento experimental inteiramente casualizado e, analisou-se número de ramos, número de botões, número de flores abertas e senescentes, altura média dos ramos, altura média das hastes florais e notas para o fechamento de vaso. Observou-se maior número e comprimento de raízes na concentração de $1.000 \mathrm{mg} \mathrm{L}^{-1}$ de AIB. A densidade de quatro plantas por vaso é recomendada para o vaso número 15.
\end{abstract}

Palavras-chave: Euryops chrysanthemoides, cobertura de vaso, densidade de plantas, floricultura.

\begin{abstract}
Rooting of cuttings and production of Euryops chrysanthemoides (DC.) B. Nord. in vase

The experiments were performed at the Department of Floriculture of UFSM from August 2013 to May 2014 in two experimental phases. The first experiment aimed to verify rooting Euryops chrysanthemoides in different concentrations of IBA, in a randomized block design, evaluated the number and length of roots. In the second experiment the different plant densities of E. chrysanthemoides pot (1, 2, 3 or 4 plants) in a completely randomized design and analyzed for the number of branches, number of buttons, number of open flowers and drought were evaluated, the average height of the branches, the middle notes of floral stems and closing the height of the vase. Observed a greater number and length of roots in $1.000 \mathrm{mg} \mathrm{L}^{-1}$ IBA. The density of four plants per pot is recommended to number 15 .
\end{abstract}

Keywords: Euryops chrysanthemoides, coverage of the vessel, plant density, floriculture.

\section{INTRODUÇÃO}

A produção comercial de flores e plantas ornamentais é uma atividade agrícola que está inserida no setor hortícola e, caracteriza-se por apresentar práticas de manejo de forma intensiva e cultivo em áreas reduzidas. Devido sua posição geográfica e diversidade climática, o Brasil torna-se um país de alto potencial na floricultura mundial, pela possibilidade de cultivar inúmeras espécies de flores e plantas ornamentais, gerando condições de obter boa colocação no mercado nacional e internacional (FERMINO e BELLÉ, 2008). Atualmente, o país cultiva de 350 espécies e aproximadamente 3000 variedades de flores e plantas ornamentais (SCHOENMAKER, 2013), resultando, no ano de 2013, a movimentação nacional desta cadeia produtiva no valor de R $\$ 5,22$ bilhões, com crescimento de $8,3 \%$ em relação ao ano de 2012 (JUNQUEIRA e PEETZ, 2014). Deste modo, a introdução de novas espécies na floricultura nacional é uma opção que pode se tornar viável.

O Euryops chrysanthemoides (DC.) B. Nord. é herbácea perene da família Asteraceae, originária da África do Sul, de arquitetura ereta e pouco ramificada, possui altura de 30 à $50 \mathrm{~cm}$ e florescimento exuberante. Folhas membranáceas, simples, profundamente divididas, com a face inferior mais clara, de 5 a $9 \mathrm{~cm}$ de comprimento. As inflorescências são em capítulos terminais e axilares, contendo flores internas reduzidas e externas de pétalas muito expandidas, ambas de cor amarela. A multiplicação da espécie ocorre, principalmente, por sementes (LORENZI, 2013). A espécie apresenta caráter ornamental, sendo muito utilizada na composição de maciços no paisagismo, contudo, sem exploração comercial em cultivo como planta envasada.

A estaquia é a técnica propagativa mais utilizada entre as culturas hortícolas, em destaque para floricultura. O emprego desta técnica é devido à capacidade de reproduzir, de forma exata, as características genéticas de qualquer planta individual (HARTMANN et al., 2002), processo no qual ocorre à indução do enraizamento adventício em segmentos destacados da planta-matriz, que, uma vez submetidos a condições favoráveis, originam uma muda (FACHINELLO et al., 2005). Visando melhor sucesso desta técnica utiliza-se o ácido indolbutírico (AIB) que é, provavelmente, a principal auxina sintética de uso geral, pois apresenta baixa toxidez para a maioria das plantas, mesmo em altas concentrações (LONE et al., 2010). Segundo Botelho et al. (2005), o AIB é efetivo para um

\footnotetext{
(1) Recebido em 13/11/14 e aceito em 21/09/15.

(2) Universidade Federal de Santa Maria, Departamento de Fitotecnia, Santa Maria-RS, Brasil. *Autor correspondente: janine_rs@hotmail.com.

(3) Engenheiro Agrônomo, Santa Maria-RS, Brasil.
} 
grande número de espécies, sendo relativamente estável e, por isso, pouco suscetível à ação dos sistemas enzimáticos de degradação das auxinas. Nas estacas tratadas com este composto, é possível encontrar resultados variáveis, consoante à espécie ou cultivar utilizada, o tipo de estaca, a época do ano, a concentração, entre outras (MACHADO et al., 2005).

O sistema de cultivo de flores em vasos visa à durabilidade do produto, em termos de qualidade. No Brasil, os critérios de comercialização de flores envasadas seguem a classificação da Cooperativa Veilling de Holambra. O padrão é determinado pela uniformidade do lote, com $90 \%$ de uniformidade quanto à altura da planta, diâmetro da planta, número de flores abertas e ponto de colheita, e, a ausência de defeitos (COOPERATIVA VEILLING DE HOLAMBRA, 2013).

Desta forma, os objetivos deste trabalho foram verificar o enraizamento de estacas de E. chrysanthemoides em diferentes concentrações de ácido indolbutírico (AIB) e avaliar diferentes densidades de plantas em vaso.

\section{MATERIAL E MÉTODOS}

Os experimentos foram realizados no período de agosto de 2013 a maio de 2014, conduzido em casa de vegetação em duas fases. O experimento 1 teve como objetivo avaliar o enraizamento de estacas de Euryops chrysanthemoides em casca de arroz carbonizada. $\mathrm{O}$ experimento 2 teve como objetivo avaliar o cultivo em vaso da mesma espécie, no Setor de Floricultura do Departamento de Fitotecnia no Campus da Universidade Federal de Santa Maria, Santa

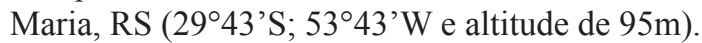

$\mathrm{O}$ experimento 1 ocorreu durante os meses de agosto a novembro de 2013. As estacas foram coletas no matrizeiro do Setor de Floricultura, da UFSM. Foram preparadas estacas com $10 \mathrm{~cm}$ de comprimento contendo 5 a 6 nós, em corte bisel (transversal), permanecendo as folhas inteiras nos nós superiores (até $3^{\circ}$ ou $4^{\circ}$ nó) e retirados os demais. Após o corte, as bases das estacas foram submetidas aos tratamentos com AIB (ácido indolbutilíco) na forma de pó, nas concentrações (zero, 250, 500 e $1.000 \mathrm{mg} \mathrm{L}^{-1}$ ). Imediatamente após o tratamento, as estacas foram alocadas em bandejas de plástico alveoladas (63 células) com volume celular $0,032 \mathrm{~L}$, contendo o substrato CAC (casca de arroz carbonizada) a profundidade de $3 \mathrm{~cm}$ da base da mesma. As bandejas foram dispostas em câmera úmida (umidade relativa de aproximadamente 80\%), no interior da casa de vegetação, com irrigações diárias, duas vezes ao dia por aspersão. Avaliaram-se o comprimento médio e o número de raízes $(\mathrm{cm})$. O experimento foi conduzido em delineamento experimental de blocos casualizados, com quatro tratamentos compostos de seis repetições, sendo que cada unidade experimental era constituída por nove estacas. Os dados foram submetidos à análise de regressão.

O experimento 2 ocorreu no período de novembro de 2013 a maio de 2014. Após a avaliação das estacas do experimento 1, as mesmas, após 90 DAP (dias após o plantio), com altura média de $8 \mathrm{~cm}$ de parte aérea e integridade radicular (torrão bem formado), foram transplantadas para vasos plásticos, de número $15(1,3 \mathrm{~L}$, $15 \mathrm{~cm}$ de diâmetro e $13 \mathrm{~cm}$ de altura) e posteriormente, foram alocados na casa de vegetação com espaçamento de 10 vasos $\mathrm{m}^{-2}$. O substrato foi constituído de três materiais, em iguais proporções de volumes, Solo São Pedro + CAC + substrato comercial H-Decker $\AA$. O experimento constituiu de cinco tratamentos com diferentes densidades e distribuição de plantas por vaso, os quais foram: T1: 1 planta por vaso; T2: 2 plantas por vaso; T3: 3 plantas dispostas em formato de triângulo por vaso; T4: 4 plantas dispostas nas bordas por vaso e T5: 4 plantas centrais com $3 \mathrm{~cm}$ entre plantas por vaso, com cinco repetições, em delineamento inteiramente casualizado. Os dados foram submetidos à análise de variância e teste $\mathrm{F}$ a $5 \%$ de probabilidade e as médias comparadas pelo teste de Tukey, a 5\% de probabilidade. As irrigações foram diárias, e a fertirrigação manejada conforme o desenvolvimento da planta, com aplicações mensais até o florescimento, após o início do florescimento as aplicações ocorreram duas vezes por semana. Foi aplicado $50 \mathrm{~mL}$ por vaso, da solução de nitrato de potássio na concentração de $\left(100 \mathrm{mg} \mathrm{L}^{-1}\right)$. Foram avaliados os parâmetros: número de flores abertas, número de flores senescentes, número de botões, número de ramos, altura média de ramos e tamanho médio da haste floral, e os vasos receberam uma para o fechamento de vaso. Adotou-se a escala de 1 a 5 (BELLÉ, 1997) que relaciona o diâmetro da planta com o diâmetro do vaso, observado em vista superior, onde a nota 1 corresponde a até $20 \%$ de fechamento de vaso; nota 2,5 a $50 \%$ de fechamento de vaso; nota 3,5 a $75 \%$ de fechamento de vaso e nota 5 a $100 \%$ de fechamento de vaso, as notas intermediárias correspondem aos intervalos percentuais de cobertura de vaso.

\section{RESULTADOS E DISCUSSÃO}

A propagação por estaquia, com o uso de AIB, da espécie Euryops chrysanthemoides, proporcionou o aumento no número de raízes, contudo, não interferiu no comprimento médio de raízes. O uso de auxinas, como o AIB, é uma prática comum, para a maximização do enraizamento de estacas de espécies ornamentais, como por exemplo, Malvaviscus arboreus (LOSS et al., 2009), Duranta repens L. (AMARAL et al., 2012) e para duas de Dendranthema grandiflora Tzvelev. cv. Stallion e cv. Toon Hermans (SCHWAB et al., 2013).

Observou-se o enraizamento de $100 \%$ as estacas em todos os tratamentos testados. O número de raízes nas estacas tratadas foi favorecido com o uso do hormônio nas concentrações de 500 a $1.000 \mathrm{mg} \mathrm{L}^{-1}$ (Figura1). O tratamento com $1.000 \mathrm{mg} \mathrm{L}^{-1}$ de AIB, obteve $35,4 \%$ a mais no número de raízes que o tratamento testemunha. Shuster et al. (2011) constataram o maior número de raízes nas estacas de Arachis pintoi Krapov. \& W. C. Greg. na concentração de $1.000 \mathrm{mg} \mathrm{L}^{-1}$ de AIB. 

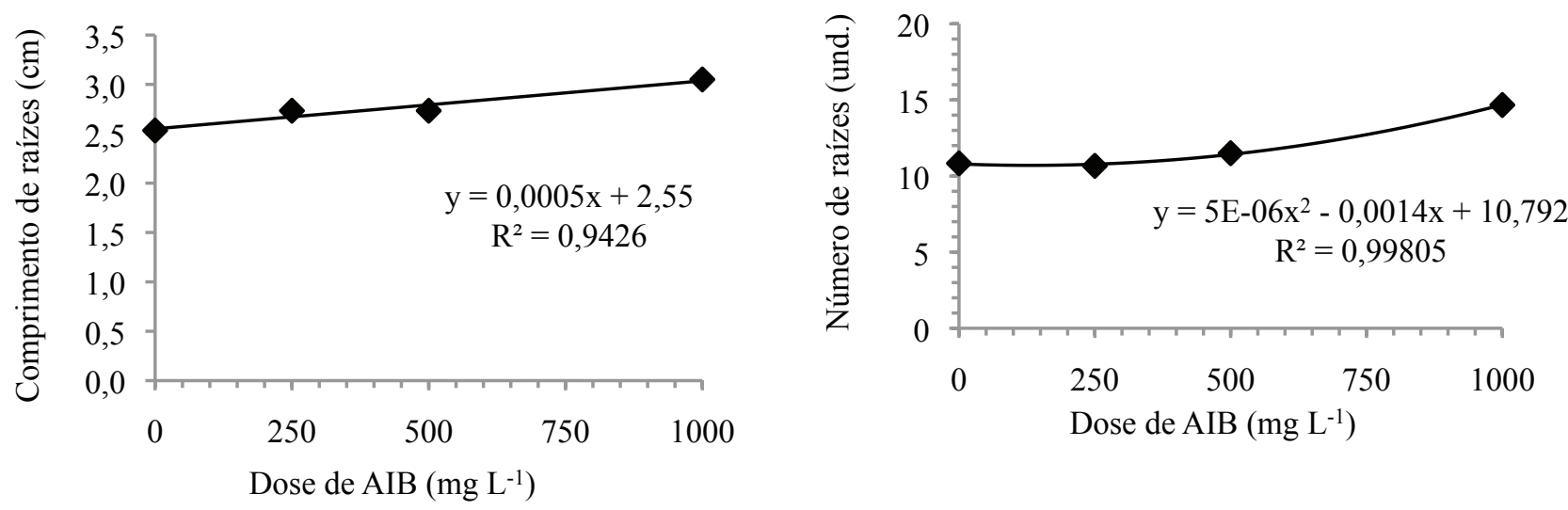

Figura 1. Estacas de Euryops chrysanthemoides sob diferentes doses de AIB (mg L-1). A: comprimento médio de raiz $(\mathrm{cm})$; B: número de raízes formadas (unidade).

Figure 1. Euryops chrysanthemoides cuttings under different doses of IBA $\left(m g L^{-1}\right)$. A: average length of root (cm); B: number of formed roots (unit)

Quanto ao comprimento médio de raíz, verificouse que houve pouca variação entre os diferentes tratamentos, observando-se uma tendência linear, isto é, houve pequeno aumento na maior dose de AIB. Maia e Botelho (2008) relataram que a aplicação de AIB, para o enraizamento de estacas de Morus nigra L., não influenciou no comprimento médio de raízes. Lone et al. (2010) não verificaram diferença no comprimento médio de raízes de estacas de Rhododendron simsii Planch. em diferentes concentrações de AIB.

Na produção de Euryops chrysanthemoides em vaso, observou-se diferença significativa entre o número de flores, abertas e senescentes, número de botões e de ramos, conforme Tabela 1. Os melhores tratamentos foram os que utilizaram quatro plantas por vaso, independente da distribuição das plantas no pote. O maior número de plantas favoreceu a maior produção de botões, flores e ramos.

Em relação à altura média dos ramos e o tamanho médio da haste floral não houve diferença significativa entre os tratamentos testados (Tabela 1), como também pode ser observado na Figura 2. Petry et al. (1999) verificaram que a densidade de planta por vaso, para o cultivo de Dendranthema grandiflora Tzvelev., não afetou a altura de plantas.

Tabela 1. Resultados médios dos diferentes tratamentos na produção de Euryops chrysanthemoides, em relação ao número de botões (BOT), número de flores abertas (FAB), número de flores senescentes (FSE), número de ramos (RAM), altura média dos ramos (AMR) e tamanho médio da haste floral (THF).

Table 1. Average of the number of buttons (BOT), number of open flowers (FAB), number of flowers senesced (ESF), the number of branches (RAM), mean height of the branches (AMR) and average size of the floral stem (THF) on different treatments for Euryops chrysanthemoides producing.

\begin{tabular}{|c|c|c|c|c|c|c|c|}
\hline \multirow{2}{*}{ Tratamento** } & BOT & FAB & FSE & RAM & AMR & THF \\
\hline & - und. - & - und. - & - und. - & - und. - & $-\mathbf{c m}-$ & $-\mathbf{c m}-$ \\
\hline 1 & $7,6 \mathrm{c}^{*}$ & $15 \mathrm{~d}$ & $8,6 \mathrm{c}$ & $34,9 \mathrm{c}$ & $23,6 \mathrm{a}$ & $15,1 \mathrm{a}$ \\
\hline 2 & $7,8 \mathrm{c}$ & $16,4 \mathrm{~cd}$ & $11 \mathrm{ab}$ & $35,2 \mathrm{c}$ & $24,7 \mathrm{a}$ & $15,7 \mathrm{a}$ \\
\hline 3 & $11,2 \mathrm{~b}$ & $18 \mathrm{bc}$ & $10,4 \mathrm{bc}$ & $39,6 \mathrm{~b}$ & $24,9 \mathrm{a}$ & $17,2 \mathrm{a}$ \\
\hline 4 & $13,8 \mathrm{a}$ & $20,6 \mathrm{ab}$ & $14,4 \mathrm{a}$ & $48,8 \mathrm{a}$ & $21,6 \mathrm{a}$ & $16,0 \mathrm{a}$ \\
\hline 5 & $14,2 \mathrm{a}$ & $21,4 \mathrm{a}$ & $14,6 \mathrm{a}$ & $50,2 \mathrm{a}$ & $15,9 \mathrm{a}$ & $15,9 \mathrm{a}$ \\
\hline $\mathrm{CV}(\%)$ & 7,92 & 7,89 & 10,52 & 3,60 & 11,74 & 9,64 \\
\hline
\end{tabular}

\footnotetext{
*As médias seguidas pela mesma letra minúscula na coluna não diferem entre si pelo teste de Tukey, p>0,05.

** Tratamento 1 (uma planta por vaso), tratamento 2 (duas plantas por vaso), tratamento 3 (três plantas por vaso, dispostas em formato de triângulo), tratamento 4 (quatro plantas por vaso, dispostas nas bordas) e tratamento 5 (quatro plantas por vaso, dispostas no centro com $3 \mathrm{~cm}$ entre as mesmas). * Means followed by the same lower case letter in the column do not differ by Tukey test, $\mathrm{p}>0.05$.

** Treatment 1 (one plant per pot) Treatment 2 (two plants per pot) Treatment 3 (three plants per pot, arranged in a triangle shape), processing four (4 plants per pot, arranged at the edges) and treating 5 (four plants per pot, placed in the center with $3 \mathrm{~cm}$ between them).
} 
As notas médias de fechamento de vaso avaliadas ao longo do desenvolvimento das plantas, aos 42, 145, 161 e 186 DAT (dias após transplante), conforme a Tabela 2. As porcentagens de fechamento de vaso foram mais amplas entre os tratamentos aos 42 DAT, atribuindose $40 ; 54,66,8 ; 78$ e $86,4 \%$ de cobertura da parte aérea sobre o vaso corresponde ao T1, T2, T3, T4 e T5, respectivamente. Isto é devido ao número de plantas por vaso, ter maior influência na cobertura do recipiente nesta fase de desenvolvimento. A partir dos 145 DAT a espécie apresentou bom desenvolvimento da parte aérea resultando no fechamento de vaso próximo a $60 \%$ (nota 3). Porcentagem considera por Bellé (1997) como apta a comercialização.

A menor diferença entre as porcentagens de cobertura de vaso foi constatada aos 186 DAT, momento este considerado próximo ao ciclo final da flor (Figura 2). Pinto et al. (2003) verificaram quando a parte aérea no vaso é bem fechada e compacta, maior a sua porcentagem de cobertura, indicando melhor o aspecto visual da planta.

Tabela 2. Evolução da porcentagem de fechamento de vaso a partir das notas atribuídas ao longo do desenvolvimento das plantas em dias após transplante (DAT).

Table 2. Evolution of the percentage of vessel closure from the grades given during the development of plants in days after transplanting (DAT).

\begin{tabular}{|c|c|c|c|c|}
\hline Tratamento & 42 DAT & $\mathbf{1 4 5}$ DAT & $\mathbf{1 6 1}$ DAT & $\mathbf{1 9 0}$ DAT \\
\hline 1 & $40 *$ & 56 & 79,2 & 92,8 \\
\hline 2 & 54 & 58,8 & 81,6 & 92 \\
\hline 3 & 68,5 & 83,5 & 91,5 & 96,5 \\
\hline 4 & 78 & 78,8 & 88,4 & 96,8 \\
\hline 5 & 86,4 & 76 & 90 & 4,38 \\
\hline
\end{tabular}

*Valores percentuais de fechamento de vaso (\%).

** tratamento 1 (uma planta por vaso), tratamento 2 (duas plantas por vaso), tratamento 3 (três plantas por vaso, dispostas em formato de triângulo), tratamento 4 (quatro plantas por vaso, dispostas nas bordas) e tratamento 5 (quatro plantas por vaso, dispostas no centro com $3 \mathrm{~cm}$ entre as mesmas).

* Percentage of vessel closure $(\%)$ values.

** Treatment 1 (one plant per pot) Treatment 2 (two plants per pot) Treatment 3 (three plants per pot, arranged in a triangle shape), processing four (4 plants per pot, arranged at the edges) and treating 5 (four plants per pot, placed in the center with $3 \mathrm{~cm}$ between them).
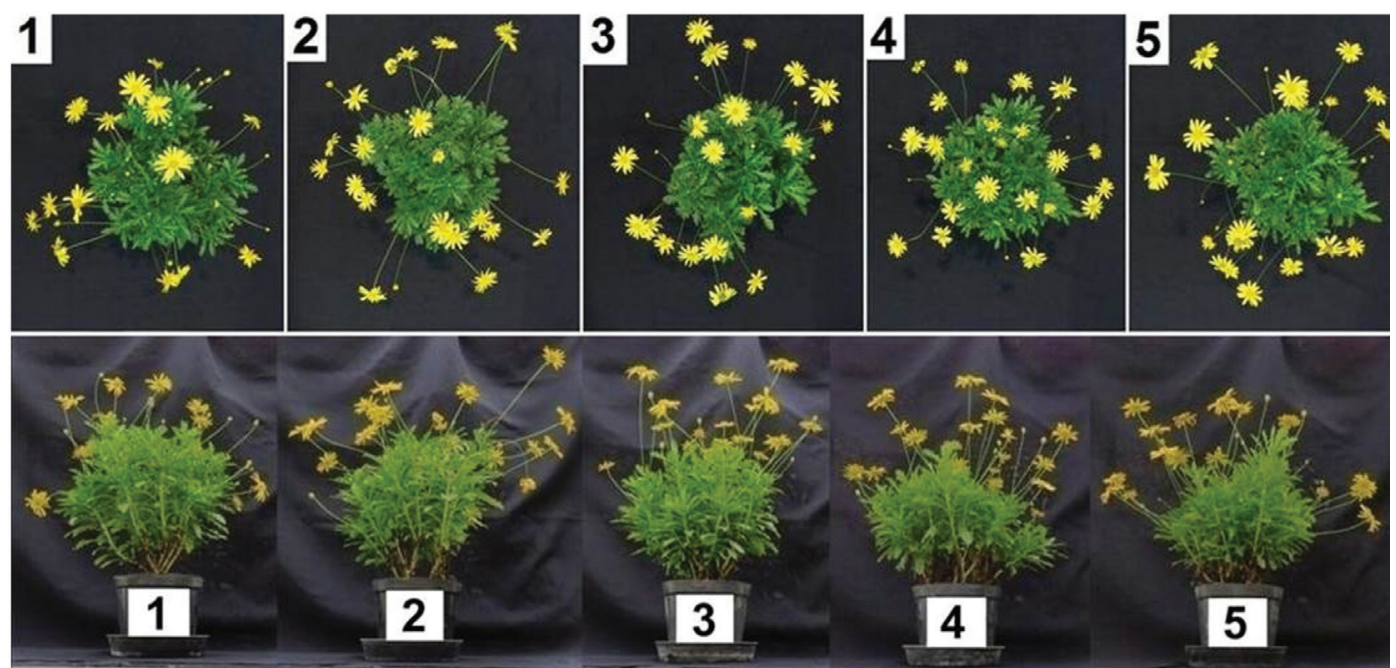

Figura 2. Plantas de Euryops chrysanthemoides cultivadas em vaso sob diferentes densidades e distribuição de plantas por vaso com 186 dias de cultivo. A: vista superior; B: vista lateral. 1: uma planta por vaso; 2: duas plantas por vaso; 3: três plantas por vaso, dispostas em formato de triângulo; 4: quatro plantas por vaso, dispostas nas bordas; 5: quatro plantas por vaso, dispostas no centro com $3 \mathrm{~cm}$ entre as mesmas. Foto: PERES, T. E. S, 2014.

Figure 2. Plants Euryops chrysanthemoides grown in pots under different densities and distribution of plants per pot with 186 days of cultivation. A: top view. B: side view. 1: one plant per pot; 2: two plants per pot; 3: three plants per pot, arranged in a triangle shape; 4: four plants per pot, placed at the edges; 5 : four plants per pot disposed in the center $3 \mathrm{~cm}$ between them. Foto: PERES, T. E. S, 2014. 


\section{CONCLUSÕES}

Conclui-se que a dose de $1.000 \mathrm{mg} \mathrm{L}^{-1}$ de ácido indolbutírico em estacas de Euryops chrysanthemoides é mais eficiente para o enraizamento das mudas.

Para o vaso número 15 recomenda-se a densidade de quatro plantas por vaso, independente da disposição das plantas, principalmente, por proporcionar resultados satisfatórios para número de botões, flores e ramos.

\section{REFERÊNCIAS}

AMARAL, G.C.; BRITO, L.P.S.; AVELINO, R.C.; SILVA JÚNIOR, J.V.; BECKMANN-CAVALCANTE, M.Z.; CAVAlCANTE, T.H.L. Produção de mudas de Duranta repens $\mathrm{L}$. pelo processo de estaquia. Revista de Ciências Agrárias, Lisboa v.35, n.1, p. 134-142, 2012.

BELLÉ, R.A. Caderno didático de floricultura. Santa Maria: Ed. UFSM. 1997. 132f.

BOTELHO, R.V.; MAIA, A.J.; PIRES, E.J.P.; TERRA, M.M.; SCHUCK, E. Efeitos de reguladores vegetais na propagação vegetativa do porta-enxerto de videira "43-43" (Vitis vinifera $\mathrm{x}$ Vitis rotundifolia). Revista Brasileira de Fruticultura, Jabuticabal, v.27, p.6-8. 2005.

COOPERATIVA VEILING DE HOLAMBRA. Critérios de comercialização. Disponível em: <www.veiling.com. br>. Acesso em: 06 jul. 2014.

FACHINELLO, J.C.; HOFFMANN, A.; NACHTIGAL, J.C. Propagação de plantas frutíferas. Brasília: EMBRAPA, 2005. 221p.

FERMINO, M.H.; BELLÉ, S. Substratos hortícolas. In: PETRY, C. (Org.). Plantas Ornamentais: aspectos para a produção. 2.ed. Passo Fundo: UPF, 2008. p.46-58.

HARTMANN, H.T.; KESTER, D.E.; DAVIES, F.T., Jr.; GENEVE, R.L. Plant propagation: principles and practices. $7^{\text {th }}$ ed. New Jersey: Prentice-Hall, 2002. 880 p.

JUNQUEIRA, A.H.; PEETZ, M.S. O setor produtivo de flores e plantas ornamentais do Brasil, no período de 2008 a 2013: atualizações, balanços e perspectivas. Revista Horticultura Ornamental, Campinas, v.20, n.2. p.115$120,2014$.

KÄMPF, A.N. Produção comercial de plantas ornamentais. Guaíba: Agropecuária, 2000. 254 p.

LONE, A.B.; LÓPEZ, E.L.; ROVARIS, S.R.S.; KLESENER, D.F.; HIGASHIBARA, L.; ATAÍDE, L.T.; ROBERTO, S.R. Efeito do AIB no enraizamento de estacas herbáceas do porta-enxerto de videira VR 4343 em diferentes substratos. Semina Ciências Agrárias, Londrina, v.31, n.3, p.599-604. 2010.
LONE, A.B.; UNEMOTO, L.K.; YAMAMOTO, L.Y.; COSTA, L.; SCHNITZER, J.A.; SATO, A.J.; RICCE, W.S.; ASSIS, A.M.; ROBERTO, S.R. Enraizamento de estacas de azaleia (Rhododendron simsii Planch.) no outono em AIB e diferentes substratos. Revista Ciência Rural, Santa Maria, v.40, n.8, p.1720-1725, 2010.

LORENZI, H.; Plantas Para Jardim no Brasil: herbáceas, arbustivas e trepadeiras. Nova Odessa: Instituto Plantarum, 2013. 373p.

LOSS, A.; TEIXEIRA, M.B.; SANTOS, T.J.; GOMES, V.M.; QUEIROZ, L.H. Indução do enraizamento em estacas de Malvaviscus arboreus Cav. com diferentes concentrações de ácido indolbutírico (AIB). Acta Scientiarum Agronomy, Maringá, v.31, n.2, p.269-273, 2009.

MACHADO, M.P.; JULIANA LISCHKA SAMPAIO MAYER, J.L.S.; RITTER, M.; BIASI, L.A. Ácido indolbutírico no enraizamento de estacas semilenhosas do porta-enxerto de videira "VR043-43" (Vitis vinifera $\mathrm{x}$ Vitis rotundifolia). Revista Brasileira de Fruticultura, Jaboticabal, v.27, p.476-479. 2005.

MAIA, A.J.; BOTELHO, R.V. Reguladores vegetais no enraizamento de estacas lenhosas da amoreira-preta cv. Xavante. Semina: Ciências Agrárias, Londrina, v.29, n.2, p.323-330, 2008.

PETRY, C.; BELEDELI, L.; ARAUJO, E.M. Respostas de cultivares de crisântemo ao manejo de iluminação e ao número de plantas por vaso. Revista Brasileira de Horticultura Ornamental, Campinas, v.5, p.121-132, 1999.

PINTO, A.C.R.; RODRIGUES, T.J.D.; LEITE, I.C.; BARBOSA, J.C. Efeitos de tamanho de vaso e sistemas de condução no desenvolvimento e qualidade de cultivares de zinia. Revista Brasileira de Horticultura Ornamental, Campinas, v.9, n.1, p.53-62, 2003.

SCHOENMAKER, K. IBRAFLOR - Release Imprensa. Instituto Brasileiro de Floricultura. Holambra. 2013. Disponível em: <http:/www.ibraflor.com/publicacoes/ vw.php?cod=213>. Acesso em: 24 jul. 2014.

SCHWAB, N.T.; NEUHAUS, M.; BELLÉ, R.A.; BACKES, F.A.A.L.; PEITER, M.X. Efeito do armazenamento a frio e desidratação sobre o enraizamento de estacas herbáceas de Dendranthema grandiflora. Revista Brasileira de Horticultura Ornamental, Campinas, v.19, n.2, p.117$120,2013$.

SHUSTER, M.Z.; SZYMCZAK, L.S.; LUSTOSA, S.B.C.; RAMALHO, K.R.O. Enraizamento de estacas de amendoim forrageiro tratadas com AIB. Revista Brasileira de Tecnologia Aplicada nas Ciências Agrárias, Guarapuava, v.4, n.2, p.122-136, 2011. 